\title{
NORMALIZATION OF WAVE FUNCTION OF THE BAND STATES
}

\author{
Abdurahim A. okHUnov ${ }^{1}$, GUlbadanbegim Sh. MAMAThonova ${ }^{2}$, Torla HJ \\ HASSAN $^{1}$, MOHAMMAD JaHANGIR AlAM ${ }^{3}$ AND MUKhTORJON X. ISMOILOV ${ }^{2}$ \\ ${ }^{I}$ Department of Science in Enginering, Faculty of Engineering, \\ International Islamic University Malaysia, \\ Jalan Gombak, 53100 Kuala Lumpur, Malaysia. \\ ${ }^{2}$ Department of of Natural Science, Margilan Academic Lyceum, Margilan, Uzbekistan. \\ ${ }^{3}$ Department of Physics, Faculty of Science, \\ International Islamic University Malaysia, Jalan Sultan Ahmad Shah, \\ Bandar Indera Mohkota, 25200 Kuantan, Pahang, Malaysia. \\ abdurahimokhun@iium.edu.my
}

(Received 17 March 2014; accepted 21 March 2015; published on line 29 May 2015)

\begin{abstract}
Low - lying band states are one of the most fundamental excitation modes in the energy spectra of deformed nuclei. This paper presents a theoretical analysis of the properties of a deformed nuclei within the phenomenological model [1]. The normalization of the wave functions of low - lying excited band states are also calculated.

ABSTRAK: Keadaan jalur aras rendah merupakan mod pengujaan paling asas dalam tenaga spekrum nuklei berbunga. Adanya kertas kerja yang menganalisa secara teori ciriciri nuklei berbunga dalam model fenomenologis [1]. Penormalan fungsi gelombang pada keadaan jalur teruja aras rendah telah dihitung.
\end{abstract}

KEYWORDS: energy; band states; nuclei; angular momentum; quantum number

\section{INTRODUCTION}

At the present time, it is observed that there is increased use of nuclear isotopes in the fields of medicine, energy and agriculture. This in turn has increased the demand for nuclear engineers. However, a more in-depth study of the structure of wave functions of nucleus and its energy states is still required. Substantial research and development of nuclear energy is still ongoing.

The description of rotational states is one of the oldest, yet not fully solved, problem in nuclear structure physics. There have been intensive experimental [2] and theoretical [3, 4] research on the structure of deformed nucleo. It is possible to note some general structure peculiarity of even-even deformed nuclei which have defined a theoretical direction to the description of experimental data. The state property of ground $(g r), \beta_{v}^{+}-$ and $\gamma_{v}^{+}$- rotational bands in even-even deformed rare-earth nuclei are influenced by the Coriolis admixtures of the states of $K^{\pi}=1_{v}^{+}$bands [5]. The cases of existence of several rotational bands with negative and positive parity are located in a very narrow interval of excited energy. This creates a prerequisite for mixing adiabatic states with the fixed values of projections of angular momentum $\mathrm{K}$, to the symmetry axis of nuclei. With growth of the angular moment I, performance of the law $E(I) \sim I(I+1)$ is violated. It can be connected 
with a change to the moment of inertia at a change of rotational frequency, and with forming of an internal excited angular momentum with a different nature.

\section{WA VE FUNCTIONS}

In the generalized model of the nucleus it is assumed that the complete nuclearHamiltonian consists of two parts:

$$
H=H\left(R^{2}\right)+H_{\mathrm{int}}, \quad \text { where } H_{\mathrm{int}}=\sum_{K} \omega_{K} b_{K}^{+} b_{K}
$$

where $H\left(R^{2}\right)$ describes the rotational energy of the nucleus and is a function of the rotational angular momentum $R(R=I+j)$. The second term is the internal part of the Hamiltonian, which we write out by introducing phonon operators $b_{K}^{+}\left(b_{K}\right)$ which create (annihilate) excited states of positive parity, and $\omega_{K}$ - are the values of the principal band energies.

From [1], the equation for a wave function that takes into account the influence Coriolis interactions, is given as:

$$
\begin{aligned}
\Psi_{M, K}^{I} & =\sum_{K^{\prime}} \Psi_{K, K^{\prime}}^{I}|I M K\rangle=\sqrt{\frac{2 I+1}{16 \pi^{2}}}\left\{\sqrt{2} \Psi_{g r, K}^{I} D_{M, 0}^{I}(\theta)\right. \\
& +\sum_{K^{\prime}} \frac{\Psi_{K^{\prime}, K}^{I}}{\sqrt{1+\delta_{K^{\prime}, 0}}}\left[D_{K, K^{\prime}}^{I}(\theta) b_{K^{\prime}}^{+}+(-1)^{I+K} D_{K,-K}^{I}(\theta) b_{-K}^{+}\right]
\end{aligned}
$$

By the theory of indignations it is possible to receive analytical expressions for the amplitude mixture $\Psi_{K, K^{\prime}}^{I}$ in Eq.1. The Hamiltonian model possesses transformation properties such that the state in Eq.1 can be classified by a quantum number, the signature $\sigma= \pm 1$, which imposes constraints on the values of the angular momentum $(-1)^{I} \sigma=1$. This leads to a significant difference on the mixing of states with a different signature.

Here we shall write the rotational part of the Hamiltonian in Eq.1 as the Taylor expansion with the power of $j$. Keeping only the zeros and first order terms, we obtain the Hamiltonian

$$
\begin{aligned}
& H=H_{r o t}\left(I^{2}\right)+H_{K, K^{\prime}}^{\sigma}(I) \\
& H_{K, K^{\prime}}^{\sigma}(I)=\omega_{K} \delta_{K, K^{\prime}}-\omega_{r o t}(I)\left(\hat{j}_{x}\right)_{K, K^{\prime}} \chi(I, K) \delta_{K, K^{\prime} \pm 1}
\end{aligned}
$$

Matrix elements from the operator $H_{c o r}$ in Eq.3 is not diagonal by basis of wave functions, while other parts of the full Hamiltonian in Eq.1 is a diagonal. The small values of angular momentum $I$ represent the small amendment to the indignant operator. In the basis state of a Hamiltonian in Eq.3, of bands with quantum numbers $K^{\pi}=0_{v}^{+}, 2_{v}^{+}$and $1_{v}^{+}$, we define amendments of the first and second order full wave functions systems with the following formula:

$$
\Psi_{M, K}^{I}=\varphi_{\tau, g r}|I M g r\rangle+\varphi_{\tau, \beta_{v}}\left|I M \beta_{v}^{+}\right\rangle+\varphi_{\tau, 1_{v}}\left|I M 1_{v}^{+}\right\rangle+\varphi_{\tau, \gamma_{v}}\left|I M \gamma_{v}^{+}\right\rangle
$$


where $\varphi_{\tau, K}$ is an amendment for states $v^{\text {th }}$ bands. This amends have following type:

For the ground bands $(v=g r)$

$$
\begin{aligned}
& \varphi_{g r, g r}=1 \\
& \varphi_{g r, \beta_{1}}=\omega_{r o t}^{2}(I) \frac{\left\langle 1\left|j_{x}\right| g r\right\rangle\left\langle 1\left|j_{x}\right| \beta_{1}\right\rangle}{\omega_{1} \varepsilon_{\beta_{1}, g r}} \\
& \varphi_{g r, \beta_{2}}=\omega_{r o t}^{2}(I) \frac{\left\langle 1\left|j_{x}\right| g r\right\rangle\left\langle 1\left|j_{x}\right| \beta_{2}\right\rangle}{\omega_{1} \varepsilon_{\beta_{2}, g r}} \\
& \varphi_{g r, 1^{+}}=\omega_{r o t}(I) \frac{\left\langle 1\left|j_{x}\right| g r\right\rangle}{\omega_{1}} \\
& \varphi_{g r, \gamma}=\omega_{r o t}^{2}(I) \frac{\left\langle 1\left|j_{x}\right| g r\right\rangle\left\langle 1\left|j_{x}\right| \gamma\right\rangle}{\omega_{1} \varepsilon_{\gamma, g r}} .
\end{aligned}
$$

For the $\beta_{1}$ bands $\left(v=\beta_{1}\right)$

$$
\begin{aligned}
& \varphi_{\beta_{1}, g r}=-\omega_{\text {rot }}^{2}(I) \frac{\left\langle 1\left|j_{x}\right| \beta_{1}\right\rangle\left\langle 1\left|j_{x}\right| g r\right\rangle}{\omega_{\beta_{1}} \varepsilon_{1, \beta_{1}}} \\
& \varphi_{\beta_{1}, \beta_{1}}=1 \\
& \varphi_{\beta_{1}, \beta_{2}}=\omega_{\text {rot }}^{2}(I) \frac{\left\langle 1\left|j_{x}\right| \beta_{1}\right\rangle\left\langle 1\left|j_{x}\right| \beta_{2}\right\rangle}{\varepsilon_{1, \beta_{1}} \varepsilon_{\beta_{2}, \beta_{1}}} \\
& \varphi_{\beta_{1}, 1^{+}}=\omega_{r o t}(I) \frac{\left\langle 1\left|j_{x}\right| \beta_{1}\right\rangle}{\varepsilon_{1, \beta_{1}}} \\
& \varphi_{\beta_{1}, \gamma}=\omega_{r o t}^{2}(I) \frac{\left\langle 1\left|j_{x}\right| \beta_{1}\right\rangle\left\langle 1\left|j_{x}\right| \gamma\right\rangle}{\varepsilon_{1, \beta_{1}} \varepsilon_{\gamma, \beta_{1}}} .
\end{aligned}
$$

For the $\beta_{2}$ bands $\left(v=\beta_{2}\right)$

$$
\begin{aligned}
& \varphi_{\beta_{2}, g r}=-\omega_{\text {rot }}^{2}(I) \frac{\left\langle 1\left|j_{x}\right| \beta_{2}\right\rangle\left\langle 1\left|j_{x}\right| g r\right\rangle}{\omega_{\beta_{2}} \varepsilon_{1, \beta_{2}}} \\
& \varphi_{\beta_{2}, \beta_{1}}=\omega_{\text {rot }}^{2}(I) \frac{\left\langle 1\left|j_{x}\right| \beta_{2}\right\rangle\left\langle 1\left|j_{x}\right| \beta_{1}\right\rangle}{\varepsilon_{1, \beta_{2}} \varepsilon_{\beta_{1}, \beta_{2}}} \\
& \varphi_{\beta_{2}, \beta_{2}}=1 \\
& \varphi_{\beta_{2}, 1^{+}}=\omega_{\text {rot }}(I) \frac{\left\langle 1\left|j_{x}\right| \beta_{2}\right\rangle}{\varepsilon_{1, \beta_{2}}} \\
& \varphi_{\beta_{2}, \gamma}=\omega_{r o t}^{2}(I) \frac{\left\langle 1\left|j_{x}\right| \beta_{2}\right\rangle\left\langle 1\left|j_{x}\right| \gamma\right\rangle}{\varepsilon_{1, \beta_{2}} \varepsilon_{\gamma, \beta_{2}}} .
\end{aligned}
$$


For the $\gamma$ bands $(v=\gamma)$

$$
\begin{aligned}
& \varphi_{\gamma, g r}=-\omega_{r o t}^{2}(I) \frac{\left\langle 1\left|j_{x}\right| \gamma\right\rangle\left\langle 1\left|j_{x}\right| g r\right\rangle}{\omega_{\gamma} \varepsilon_{1, \gamma}} \\
& \varphi_{\gamma, \beta_{v}}=-\omega_{r o t}^{2}(I) \frac{\left\langle 1\left|j_{x}\right| \gamma\right\rangle\left\langle 1\left|j_{x}\right| \beta_{v}\right\rangle}{\varepsilon_{1, \gamma} \varepsilon_{\gamma, \beta_{v}}} \\
& \varphi_{\gamma, 1^{+}}=\omega_{r o t}(I) \frac{\left\langle 1\left|j_{x}\right| \gamma\right\rangle}{\varepsilon_{1, \gamma}} \\
& \varphi_{\gamma, \gamma}=1 .
\end{aligned}
$$

where $\varepsilon_{v, \alpha}=\omega_{v}-\omega_{\alpha}$ and $\left\langle 1\left|\hat{j}_{x}\right| K\right\rangle-$ matrix elements of Coriolis mixture of states $K^{\pi}=0_{v}^{+}, 2_{v}^{+}$and $1_{v}^{+}$band.

Taking into account normalizations for Coriolis interactions we have

$$
\Psi_{\tau, \alpha}^{I}=\frac{\varphi_{\tau, K}^{I}}{N_{\tau}^{I}}, \quad N_{\tau}^{I}=\sqrt{\sum_{1}\left(\varphi_{\tau, 1}^{I}\right)^{2}}
$$

We can see from the formulas in Eq.5 to Eq.8, if a value of matrix element is large (Table 1) and headband levels are located close, there is a hybridization of a band, which is strongly shown in values of probabilities of transitions (feature work).

Table 1: The matrix elements of the Coriolis interaction $\left(j_{x}\right)_{K, K^{\prime}}$ for isotopes ${ }^{170,172,174} \mathrm{Yb}$ correspondingly

\begin{tabular}{cccccc}
\hline $\mathrm{A}$ & $\left(j_{x}\right)_{g r, 1}$ & $\left(j_{x}\right)_{\beta_{1}, 1}$ & $\left(j_{x}\right)_{\beta_{2}, 1}$ & $\left(j_{x}\right)_{\beta_{3}, 1}$ & $\left(j_{x}\right)_{\gamma_{1}, 1}$ \\
\hline${ }^{170} Y \mathrm{~b}$ & 0.1864 & 0.3936 & 0.6586 & 0.9081 & 0.7278 \\
${ }^{172} \mathrm{Yb}$ & 0.2457 & 0.9777 & 0.7176 & 0.1100 & 0.3250 \\
${ }^{174} \mathrm{Yb}$ & 0.1850 & 0.4000 & 0.2500 & 0.1500 & 0.0850 \\
\hline
\end{tabular}

\section{RESULTS}

The Coriolis mixture coefficients $\Psi_{K, K^{\prime}}^{I}$ for the states of the ground band state, $\beta_{v}^{+}-$, $\gamma_{v}^{+}-$and $1_{v}^{+}$bands for isotope ${ }^{170} \mathrm{Yb}$ is illustrated in Fig. 1, taken by the description energy of spectra states. We see that even in a low value of spin $\beta_{1}^{+}$and $1_{v}^{+}$there is a strong mixture of components in $\gamma_{1}^{+}$band states. This is observed from the closely arranged energy bands, which shows that the value lies within the probability of electromagnetic transitions.

From Fig. 2 we can see that there is a strong mixture in the $\beta_{1}\left(K^{\pi}=0_{2}^{+}\right)$and $\gamma_{1}\left(K^{\pi}=2_{2}^{+}\right)$band state in ${ }^{170} Y b$, and also $\beta_{2}\left(K^{\pi}=0_{3}^{+}\right)$and $\gamma_{1}\left(K^{\pi}=2_{2}^{+}\right)$band state in ${ }^{172} \mathrm{Yb}$, even for low spin values of $I$. 


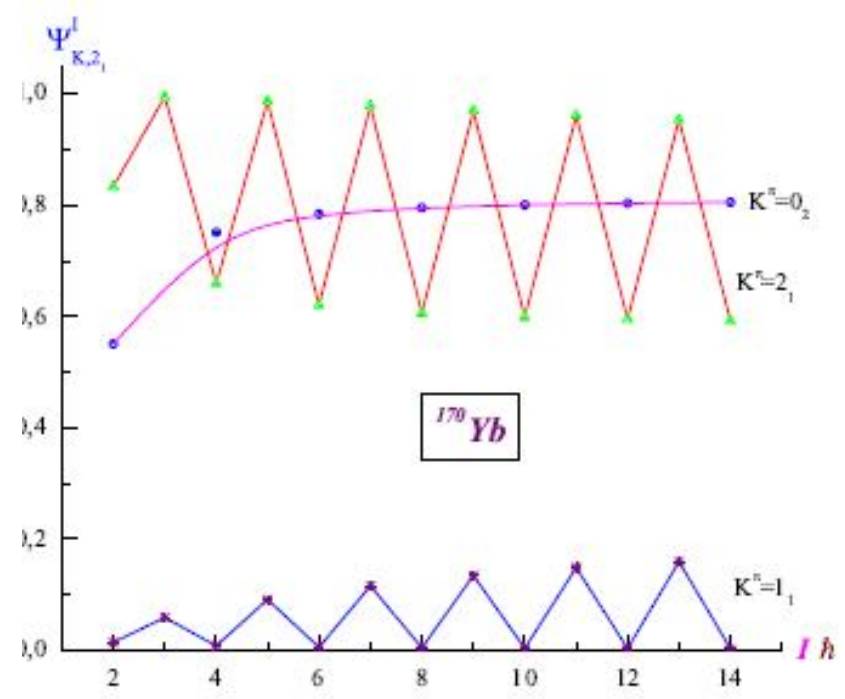

Fig. 1 The mixture coefficients of ${ }^{170} \mathrm{Yb}$ isotope.

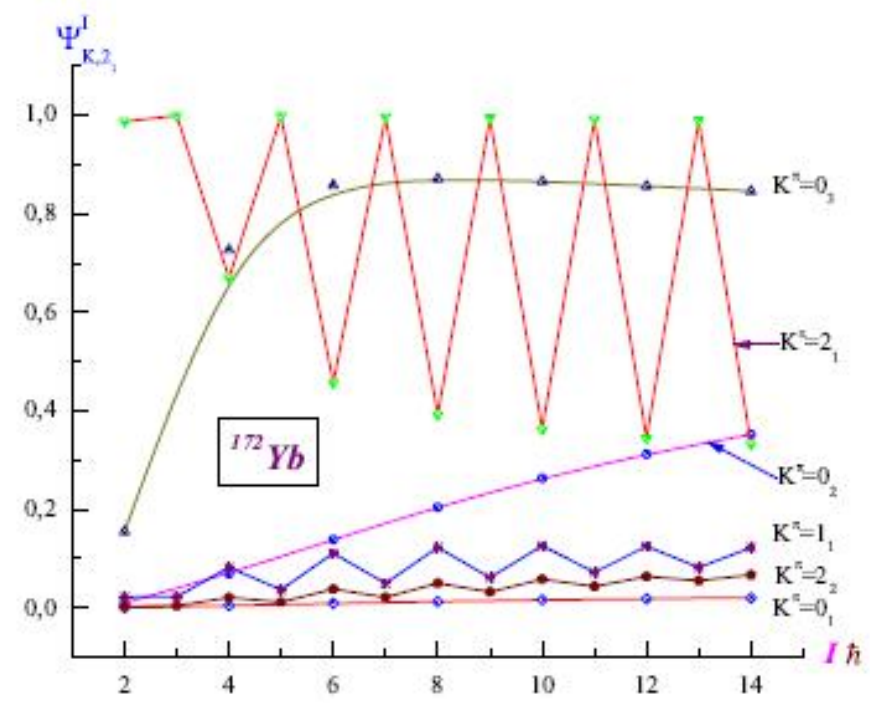

Fig. 2 The mixture coefficients of ${ }^{172} Y b$ isotope.

\section{CONCLUSION}

A theoretical framework was introduced in the paper. To understand structure properties of states, we use the phenomenological model [1], which clearly describes large number of experimental data by the deviation properties of the positive parity in even-even deformed nuclei from the role of adiabatic theory. The mixture components of the band states are analyzed for the $g r, \beta_{v}^{+}-, \gamma_{v}^{+}-$and $K^{\pi}=1^{+}$rotational bands of the isotopes $170,172,174 \mathrm{Yb}$ respectively.

\section{ACKNOWLEDGEMENT}

The work here is supported by Grant EDW13-034-0919, International Islamic University Malaysia and the Margilan Academic Lyceum. 


\section{REFERENCES}

[1] Usmanov P.N, Okhunov A.A, SAlikhbaev U.S, Vdovin A. I. (2010) Analysis of Electromagnetic Ttransitions in Nuclei ${ }^{176,178}$ Hf. Phys. Part. Nucl., 7(3):185-191

[2] Leander GA. (1985) in Nuclear Structure, eds. Broglia RA, Hagemann GB, Herskind B, North-Holland, Amsterdam, pp. 249.

[3] Mikhaylov IN, Usmanov Ph.N, Okhunov AA, Briancon Sh. (1992) Quadruple electrical transitions in isotopes ${ }^{156,158,160,162,164}$ Dy. Izv AS USSR, ser. Fiz., 56(1):121-128.

[4] Gromov KYa, Usmanov Ph.N, Xolmatov A.X, Butabaev Yu.S, Niyazov R. A. (1993) Nonadiabaticity of monopole and quadrupole transitions of 156Gd Yadernay Fizika, 56(12): p.28

[5] Bohr A, Mottelson B. (1997) Nuclear Structure, Vvol. 1,2 (New York: Benjamin)

[6] Usmanov Ph.N, Mikhailov IN. (1997) Effects of nonadiabaticity of collective motion in even-even deformed nuclei. Phys. Part. Nucl. 28(4):348-373

[7] Fahlander C, Varnestig B, Bäcklin F., Svensson L.E., Disdier D., Kraus L., Linck I., Schulz N. Pedersen J. (1992) Coulomb excitation of ${ }^{172}$ Yb. Nucl. Phys., A147:157-172

\section{NOMENCLATURE}

\section{i) Greek letters}

$\beta \quad$ Nuclear Bands (energy level band)

$\gamma \quad$ Nuclear Bands (energy level band)

$\varphi \quad$ Wave function

ii) Subscript

I Angular momentum

$K \quad$ Quantum number

$H \quad$ Hamiltonian 